\title{
Arborização urbana no Município de Três Rios, Rio de Janeiro, Brasil
}

\section{Izabela Cristina Moreira Moraes $^{1}$ e Michaele Alvim Milward-de-Azevedo}

\author{
${ }^{1}$ Universidade do Estado do Rio de Janeiro. Programa de Pós-Graduação em \\ Biologia Vegetal. Rua São Francisco Xavier, 524. Maracanã. Rio de Janeiro-RJ, Brasil \\ (CEP 20550-900). \\ ${ }^{2}$ Universidade Federal Rural do Rio de Janeiro. Instituto Três Rios. Departamento \\ de Ciências do Meio Ambiente. Av. Prefeito Alberto da Silva Lavinas, 1847. Centro. \\ Três Rios-RJ, Brasil (CEP 25802-100). E-mail: michaelemilward@gmail.com.
}

Resumo. A arborização urbana é utilizada para minimizar problemas acarretados pela urbanização. Três Rios, município localizado no estado do Rio de Janeiro, apresenta um elevado processo de desenvolvimento, necessitando de um planejamento adequado para estar em equilíbrio com o meio ambiente. 0 objetivo deste estudo foi realizar uma análise quantitativa e qualitativa da diversidade de árvores utilizadas na arborização urbana de Três Rios. O levantamento florístico foi executado em três logradouros de cinco bairros distintos e os parâmetros fitossociológicos e o índice de diversidade de espécies foram calculados. Foram amostrados 452 indivíduos pertencentes a 19 famílias e 45 espécies, sendo 62\% exóticas do Brasil e 38\% nativas. As famílias Fabaceae e Malvaceae apresentaram o maior número de espécies. A espécie mais representativa foi Terminalia catapa. Foi constatado que $38 \%$ dos indivíduos apresentam interferência na rede elétrica e $30 \%$ nas calçadas. Três Rios não possui um planejamento urbano que aborde a arborização, visto que o inventário demonstrou uma grande proporção de espécies exóticas com muitos indivíduos que sobrepõe à proporção de espécies nativas. Outro fator que também demonstra a falta de planejamento foi à incompatibilidade com pavimento e rede elétrica, onde grande parte dos indivíduos arbóreos encontra-se em conflito, oferecendo riscos à população.

Palavras-chave: Conservação; Espécies exóticas; Levantamento florístico; Planejamento urbano.

\section{Abstract. Urban afforestation in Municipality of Três Rios, Rio de Janeiro, Brazil. Urban afforestation is utilized to downplay urban-related problems. Três Rios, a municipality located in Rio de Janeiro State, presents an elevated process of development, requiring a suitable planning in order to be in balance with the environment. The objective of this study was to analyze, in qualitative and quantitative terms, the diversity of trees used in}

Recebido 29/07/2020

Aceito $15 / 04 / 2021$

Publicado $30 / 04 / 2021$

Acesso aberto

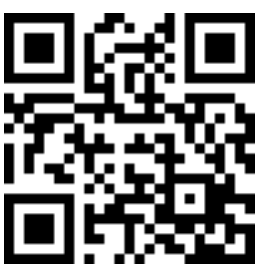

(D) $0000-0002-5542-747 \mathrm{X}$ Izabela Cristina Moreira Moraes 0000-0001-8076-5561 Michaele Alvim Milward-de-Azevedo

ISSN 2359-1412/RBGAS-2020-0169/2021/8/18/28/433

Rev. Bras. Gest. Amb. Sustent.

http://revista.ecogestaobrasil.net 
Três Rios urban afforestation. The floristic data gathering was executed in three places among five distant neighborhoods. It was sampled 452 individuals belonging to 19 families and 45 species, while $62 \%$ are exotic and $38 \%$ native of Brazil. The families Fabaceae and Malvaceae present the largest number of species. The species most representative was Terminalia catapa. It was verified that $38 \%$ of the individuals present interference in the power grids and $30 \%$ on the sidewalks. Três Rios does not posses an urban plan that integrates urban afforestation, since the inventory indicated that a large portion of the exotic species, with lots of individuals that overlap the native species. Another factor that also indicates the lack of planning was the incompatibility with the sidewalk and the electric grid, in which a big part of arboreal individuals find themselves in conflict, and therefore offering risks to the population.

Keyword: Conservation; Exotic species; Florist survey; Urban planning.

\section{Introdução}

O processo de urbanização se encontra cada vez mais crescente, e aliado a este contínuo desenvolvimento é perceptível a ocorrência de extremas modificações na paisagem natural (Melo e Piacentini, 2011), resultando em perda de fragmentos de vegetação nativa, destruição de paisagens naturais, bruscas mudanças no ambiente, e influenciando negativamente na qualidade de vida da população.

Com isso, torna-se cada vez mais emergente a necessidade de implementação de áreas verdes nos espaços públicos, além da inclusão de projetos que visem a sustentabilidade nos planejamentos urbanos, a fim de se alcançar condições ideais de vivência (Carneiro e Carvalho, 2013). A arborização urbana das vias públicas é uma estratégia utilizada para minimizar tais problemas (Faria et al., 2007), acarretados pela urbanização, caracterizando-se como forma complementar de conservação de espécies.

O planejamento prévio da arborização urbana avalia as características das espécies à serem implantadas estão em consonância com as estruturas municipais, garantindo os benefícios advindos da arborização, sem que haja conflitos futuros com os elementos que compõem o ambiente construído (Iwama, 2014). A falta de um planejamento pode ocasionar no uso excessivo de espécies exóticas, pois após serem introduzidas em um ambiente, estas têm a capacidade de se estabelecer, desenvolver populações autoregenerativas e dominar o espaço de espécies nativas da região, causando assim, impactos ambientais (Blum et al., 2008).

Essas espécies são reconhecidas como a segunda maior causa de extinção de espécies no planeta, tendo em vista seu poder de alteração dos processos ecossistêmicos e consequentes mudanças no seu funcionamento, através da contaminação biológica (Sampaio et al., 2011), afetando diretamente na biodiversidade, economia e saúde humana.

No Brasil, a grande maioria das cidades não possui planejamento urbano que abarque a arborização (Graciano-Silva, 2014), o que reflete em uma arborização caracterizada como inadequada, pouco diversificada, descontínua e comumente composta por espécies exóticas (Melo e Piacentini, 2011).

O Município de Três Rios, localizado no Estado do Rio de Janeiro, na Região CentroSul Fluminense, às margens do Rio Paraíba do Sul, apresenta um elevado processo de 
desenvolvimento econômico e social, atraindo muitas indústrias e proporcionando a intensa expansão urbana (Candido, 2015). Necessitando de um planejamento adequado para estar em equilíbrio com o meio ambiente e garantir uma boa qualidade de vida para seus habitantes, porém, este não foi abordado no plano diretor do município, revisado pela Lei no 3.906/2013 (Três Rios, 2013).

Logo, este trabalho teve como objetivo analisar a diversidade de árvores utilizadas na arborização urbana de Três Rios, caracterizando de forma quantitativa e qualitativa a arborização das vias públicas, avaliando à questão das espécies exóticas invasoras e a relação dos indivíduos arbóreos com as estruturas urbanas, tais como rede elétrica, calçadas e muros.

\section{Material e métodos}

O Município de Três Rios (Figura 1) está localizado na Mesorregião Centro-Sul Fluminense, entre as coordenadas geográficas de referência $22^{\circ} 06^{\prime} 58^{\prime \prime} \mathrm{S}$ e $43^{\circ} 12^{\prime} 23^{\prime \prime} \mathrm{O}$, no Estado do Rio de Janeiro (IBGE, 2014). Apresenta uma área de $326,136 \mathrm{~km}^{2}$ e uma população de 102.212 habitantes, com uma parcela flutuante girando em torno de $400 \mathrm{mil}$ pessoas, por ser uma cidade-pólo (IBGE, 2014). De acordo com Silva-Filho et al. (2013), a cidade apresenta um clima mesotérmico, com verão quente e chuvoso, e temperatura variando de $14,2{ }^{\circ} \mathrm{C}$ até $37,4{ }^{\circ} \mathrm{C}$. A vegetação é composta por Floresta Estacional Semidecidual com solos do tipo argiloso (IBGE, 2012).

O levantamento florístico da arborização urbana foi realizado entre setembro de 2018 e maio de 2019, em cinco bairros Cantagalo, Centro, Nova Niterói, Palmital e Triângulo (Figura 1). Em cada bairro, foram analisados três logradouros, com exceção do bairro Nova Niterói, com apenas dois logradouros. Cantagalo: Rua Maestro Costa Barros (300 m), Rua Benjamin Constant (800 m) e Praça JK (300 m); Centro: Rua 15 de Novembro (700 m), Rua Padre Conrado (130 m) e Rua Barão de Entre Rios (700 m); Nova Niterói: Avenida Arariboia $(1.800 \mathrm{~m}$ ) e Praça Cacique $(85 \mathrm{~m})$; Palmital: Rua José Martins Costa (130 m), Rua Remo Righi (350 m) e Rua Miguel da Silva (240 m); Triângulo: Praça Dario de Moraes (185 m), Praça Zaquieu Esmeralda (189 m) e Avenida do Contorno (1.100 m).

As árvores avaliadas apresentaram CAP (circunferência a altura do peito, medida a $1,30 \mathrm{~m}$ do solo) igual ou maior que $15 \mathrm{~cm}$, as quais foram coletadas informações qualitativas dos indivíduos arbóreos, espécie, família, CAP, altura total e interferência nos elementos urbanos (muro, fiação elétrica e calçada).

Os nomes das espécies foram atualizados de acordo com o sistema de classificação atual APG IV (2016), e com auxílio do projeto Flora do Brasil 2020, do Jardim Botânico do Rio de Janeiro (http://floradobrasil.jbrj.gov.br), para as espécies brasileiras, e do "The Plant List" (http://www.theplantlist.org), para as espécies exóticas.

A fim de dimensionar as proporções das espécies utilizadas na arborização, as espécies foram categorizadas de acordo com as procedências geográficas: Exótica dos Ecossistemas Brasileiros (EX-BR) - não ocorre espontaneamente nos ecossistemas brasileiros; Nativa Brasileira (NBR) - ocorre no Brasil; Exótica da Mata Atlântica (EX-MA) não ocorre espontaneamente no ecossistema Mata Atlântica; Nativa da Mata Atlântica (NMA) - ocorre naturalmente no ecossistema Mata Atlântica. 

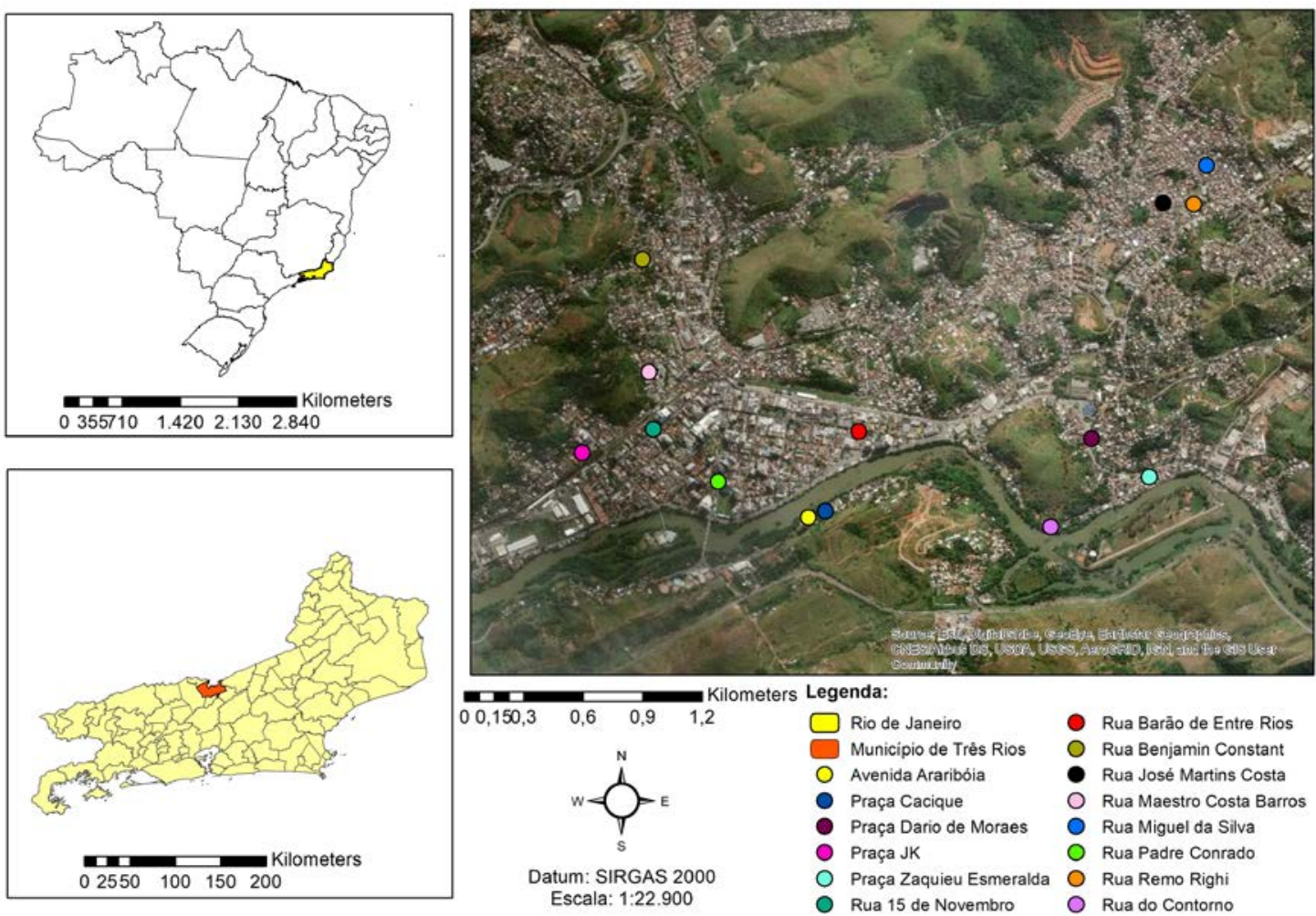

Figura 1. Localização do Município de Três Rios, Rio de Janeiro, Brasil, e indicação de ruas avaliadas no levantamento da arborização urbana.

Os parâmetros fitossociológicos foram calculados segundo Mueller-Dombois e Ellenberg (1974), através do programa FITOPAC 2.1 (Shepherd, 2009). O Coeficiente de Mistura de Jentsch (QM) (Hosokawa, 1981) também foi utilizado para calcular a diversidade da composição da arborização urbana levantada, apontando o número de árvores de cada espécie que é encontrado na área de estudo, medindo a intensidade de mistura das espécies e os possíveis problemas de manejo.

\section{Resultados e discussão}

Foram amostrados 452 indivíduos pertencentes a 19 famílias e 45 espécies arbustivas e arbóreas (Tabela 1). Dentre as 45 espécies encontradas na arborização urbana de Três Rios, 62\% (28) são exóticas do Brasil e 38\% (17) são nativas brasileiras, destas, quatro não ocorrem no bioma Mata Atlântica (Tabela 1).

De acordo com o levantamento realizado, apenas oito famílias e 13 espécies são nativas da Mata Atlântica, destas, 11 espécies ocorrem no Estado do Rio de Janeiro (Tabela 1). As espécies Licania tomentosa (Benth.) Fritsch (Chrysobalanaceae - oiti) e Plinia cauliflora (Mart.) Kausel (Myrtaceae - jabuticaba) são endêmicas da Mata Atlântica, sendo que Licania tomentosa não ocorre na Região Sudeste do Brasil, e apresenta a maior representação entre os indivíduos nativos da Mata Atlântica, na arborização urbana de Três Rios. Faria et al. (2013) e Bastos et al. (2016) mencionaram também uma grande quantidade de indivíduos desta espécie em outras ruas de Três Rios. Em contrapartida, Plinia cauliflora está representada por apenas um indivíduo. 
Tabela 1. Lista das espécies presentes na arborização urbana do Município de Três Rios, Rio de Janeiro, Brasil.

\begin{tabular}{|c|c|c|c|c|c|}
\hline Família (espécies) & Nome popular & $\begin{array}{c}\text { № de } \\
\text { indivíduos }\end{array}$ & Origem & $\begin{array}{l}\text { Ocorrência } \\
\text { no RJ }\end{array}$ & $\begin{array}{c}\text { Interferência } \\
\text { trânsito de } \\
\text { pedestres }\end{array}$ \\
\hline \multicolumn{6}{|l|}{ Anacardiaceae } \\
\hline Mangifera indica L.* & Mangueira & 14 & EX-BR & & Sim \\
\hline Schinus terebinthifolia Raddi* & $\begin{array}{c}\text { Pimenta-rosa/ } \\
\text { aroeira }\end{array}$ & 2 & $\begin{array}{l}\text { NBR/ } \\
\text { NMA }\end{array}$ & $\mathrm{X}$ & Não \\
\hline \multicolumn{6}{|l|}{ Apocynaceae } \\
\hline Nerium oleander L. & Oleandro & 5 & EX-BR & & Não \\
\hline Plumeria rubra L. & Jasmim-manga & 1 & EX-BR & & Não \\
\hline \multicolumn{6}{|l|}{ Araucariaceae } \\
\hline $\begin{array}{l}\text { Araucaria columnaris (G. Forst.) } \\
\text { Hook }\end{array}$ & $\begin{array}{l}\text { Pinheiro-de- } \\
\text { natal } \\
\end{array}$ & 1 & EX-BR & & Não \\
\hline \multicolumn{6}{|l|}{ Arecaceae } \\
\hline Cocos nucifera L. & Coqueiro & 3 & $\begin{array}{l}\text { NBR/ } \\
\text { NMA }\end{array}$ & $\mathrm{X}$ & Sim \\
\hline \begin{tabular}{lll|}
$\begin{array}{l}\text { Socratea exorrhiza } \\
\text { Wendl. }\end{array}$ & & \\
Wart.) & H. \\
\end{tabular} & $\begin{array}{l}\text { Palmeira- } \\
\text { andante }\end{array}$ & 4 & $\begin{array}{c}\text { NBR/ } \\
\text { EX-MA }\end{array}$ & & Não \\
\hline $\begin{array}{l}\text { Syagrus romanzoffiana (Cham.) } \\
\text { Glassman }\end{array}$ & Jerivá & 26 & $\begin{array}{l}\mathrm{NBR} / \mathrm{N} \\
\mathrm{MA}\end{array}$ & $X$ & Não \\
\hline \multicolumn{6}{|l|}{ Asparagaceae } \\
\hline Dracaena fragrans (L.) Ker Gawl. & Dracena & 3 & EX-BR & & Não \\
\hline \multicolumn{6}{|l|}{ Bignoniaceae } \\
\hline $\begin{array}{l}\text { Handroanthus chrysotrichus } \\
\text { (Mart. ex DC.) Mattos* }\end{array}$ & Ipê-amarelo & 3 & $\begin{array}{l}\text { NBR/N } \\
\text { MA }\end{array}$ & $\mathrm{X}$ & Não \\
\hline $\begin{array}{l}\text { Handroanthus impetiginosus } \\
\text { (Mart. ex DC.) Mattos }\end{array}$ & $\begin{array}{l}\text { Ipê-rosa / Ipê- } \\
\text { roxo }\end{array}$ & 24 & $\begin{array}{c}\mathrm{NBR} / \mathrm{N} \\
\mathrm{MA}\end{array}$ & $\mathrm{X}$ & Sim \\
\hline $\begin{array}{l}\text { Handroanthus serratifolius (Vahl) } \\
\text { S. Grose* }\end{array}$ & Ipê-amarelo & 1 & $\begin{array}{c}\mathrm{NBR} / \mathrm{N} \\
\mathrm{MA}\end{array}$ & $\mathrm{X}$ & Não \\
\hline Tecoma stans (L.) Juss. ex Kunth* & Ipê-de-jardim & 3 & EX-BR & & Não \\
\hline \multicolumn{6}{|l|}{ Caricaceae } \\
\hline Carica papaya L. & Mamão & 1 & EX-BR & & Não \\
\hline \multicolumn{6}{|l|}{ Chrysobalanaceae } \\
\hline $\begin{array}{lll}\text { Licania } \quad \text { tomentosa } & \text { (Benth.) } \\
\text { Fritsch* } & & \\
\end{array}$ & Oiti & 39 & NMA & & Sim \\
\hline \multicolumn{6}{|l|}{ Combretaceae } \\
\hline Terminalia catappa L. * & Amendoeira & 50 & EX-BR & & Sim \\
\hline \multicolumn{6}{|l|}{ Fabaceae } \\
\hline Albizia lebbeck (L.) Benth.* & $\begin{array}{l}\text { Coração-de- } \\
\text { negro }\end{array}$ & 7 & EX-BR & & Sim \\
\hline Bauhinia blakeana Dunn & Árvore-orquídea & 1 & EX-BR & & Não \\
\hline Bauhinia variegata L.* & Pata-de-vaca & 23 & EX-BR & & Sim \\
\hline $\begin{array}{l}\text { Bauhinia variegata var. candida } \\
\text { Voigt }\end{array}$ & $\begin{array}{l}\text { Pata-de-vaca- } \\
\text { branca } \\
\end{array}$ & 1 & EX-BR & & Sim \\
\hline Cassia fistula L. & Chuva-de-ouro & 15 & EX-BR & & Sim \\
\hline $\begin{array}{lll}\text { Clitoria fairchildiana } & \text { R. } & \text { A. } \\
\text { Howard* } & & \\
\end{array}$ & Sombreiro & 2 & $\begin{array}{c}\text { NBR/N } \\
\text { MA }\end{array}$ & $X$ & Não \\
\hline $\begin{array}{l}\text { Delonix regia (Bojer ex Hook.) } \\
\text { Raf. (Bojer ex Hook.) Raf.* }\end{array}$ & Flamboyant & 9 & EX-BR & & Sim \\
\hline $\begin{array}{l}\text { Leucaena leucocephala (Lam.) De } \\
\text { Wit.* }\end{array}$ & Leucena & 27 & EX-BR & & Não \\
\hline
\end{tabular}


Tabela 1. Continuação.

\begin{tabular}{|c|c|c|c|c|c|}
\hline Família (espécies) & Nome popular & $\begin{array}{c}\text { № de } \\
\text { indivíduos }\end{array}$ & Origem & $\begin{array}{l}\text { Ocorrência } \\
\text { no RJ }\end{array}$ & $\begin{array}{c}\text { Interferência } \\
\text { trânsito de } \\
\text { pedestres }\end{array}$ \\
\hline $\begin{array}{l}\text { Peltophorum dubium (Spreng.) } \\
\text { Taub. }\end{array}$ & Angico-amarelo & 8 & $\begin{array}{c}\text { NBR/N } \\
\text { MA }\end{array}$ & $\mathrm{X}$ & Não \\
\hline Senna siamea Lam.* & Cássia-Sião & 22 & EX-BR & & Sim \\
\hline \multicolumn{6}{|l|}{ Lythraceae } \\
\hline Lagerstroemia indica L.* & Resedá & 8 & EX-BR & & Não \\
\hline $\begin{array}{l}\text { Physocalymma } \\
\text { Pohl. }\end{array}$ & Pau-de-rosas & 1 & $\begin{array}{l}\text { NBR/E } \\
\text { X-MA }\end{array}$ & & Não \\
\hline \multicolumn{6}{|l|}{ Magnoliaceae } \\
\hline $\begin{array}{l}\text { Magnolia champaca (L.) Baill. ex } \\
\text { Pierre }\end{array}$ & $\begin{array}{l}\text { Magnólia- } \\
\text { amarela }\end{array}$ & 1 & EX-BR & & Não \\
\hline \multicolumn{6}{|l|}{ Malpighiaceae } \\
\hline Malpighia emarginata DC. & Acerola & 3 & EX-BR & & Não \\
\hline \multicolumn{6}{|l|}{ Malvaceae } \\
\hline \begin{tabular}{lll|} 
Ceiba speciosa & (A. & St.-Hil.) \\
Ravenna* & & \\
\end{tabular} & Paineira-rosa & 3 & $\begin{array}{l}\mathrm{NBR} / \mathrm{N} \\
\mathrm{MA}\end{array}$ & $\mathrm{X}$ & Não \\
\hline Dombeya acutangula Cav. & Astrapéia-rosa & 1 & EX-BR & & Não \\
\hline Hibiscus rosa-sinensis L. & Hibisco & 1 & EX-BR & & Não \\
\hline $\begin{array}{l}\text { Ochroma pyramidale (Cav. ex } \\
\text { Lam.) Urb. }\end{array}$ & Pau de balsa & 1 & $\begin{array}{l}\text { NBR/E } \\
\mathrm{X}-\mathrm{MA}\end{array}$ & & Não \\
\hline Pachira aquatica Aubl.* & Munguba & 37 & $\begin{array}{l}\text { NBR/E } \\
\text { X-NMA }\end{array}$ & & Sim \\
\hline Guazuma ulmifolia Lam. & Fruta-de-macaco & 1 & $\begin{array}{l}\text { NBR/N } \\
\text { MA }\end{array}$ & $\mathrm{X}$ & Não \\
\hline \multicolumn{6}{|l|}{ Moraceae } \\
\hline Morus nigra L.* & Amoreira-preta & 3 & EX-BR & & Sim \\
\hline Ficus benjamina L. & Ficus & 21 & EX-BR & & Sim \\
\hline \multicolumn{6}{|l|}{ Myrtaceae } \\
\hline Plinia cauliflora (Mart.) Kausel & Jabuticaba & 1 & NMA & $\mathrm{X}$ & Não \\
\hline Psidium guajava L.* & Goiabeira & 7 & EX-BR & & Sim \\
\hline Syzygium cumini (L.) Skeels* & Jamelão & 7 & EX-BR & & Não \\
\hline Syzygium jambos (L.) Alston & Jambo & 5 & EX-BR & & Sim \\
\hline \multicolumn{6}{|l|}{ Oleaceae } \\
\hline Ligustrum lucidum W. T. Aiton* & Alfeneiro & 7 & EX-BR & & Não \\
\hline \multicolumn{6}{|l|}{ Poligygonaceae } \\
\hline Triplaris americana L.* & Pau-formiga & 3 & $\begin{array}{l}\text { NBR/N } \\
\text { MA }\end{array}$ & & Não \\
\hline \multicolumn{6}{|l|}{ Rutaceae } \\
\hline Murraya paniculata (L.) Jack & Murta & 36 & EX-BR & & Não \\
\hline
\end{tabular}

Legenda: Origem - EX-BR: exótica do Brasil, EX-MA: exótica da Mata Atlântica, NBR: nativa do Brasil, NMA: nativa da Mata Atlântica; RJ: ocorre no Estado do Rio de Janeiro; *espécies encontradas no levantamento florístico da mata ciliar urbana do Rio Paraíba do Sul no Município de Três Rios (Cândido, 2015; Cândido et al., 2018).

A ampla utilização de espécies exóticas na arborização urbana no Brasil é comum, e pode ser evidenciado em levantamentos da arborização urbana no Brasil. A utilização de espécies exóticas na arborização urbana influencia na perda da diversidade local, ocupando o espaço das nativas, em remanescentes florestais, além de serem vistas pela 
população como espécies nativas da região, devido à sua extensa utilização na arborização (Blum et al. 2008).

As Famílias Fabaceae (10 ssp., 22,2\%) e Malvaceae (6 ssp., 13,3\%) apresentam-se com o maior número de espécies, e dez famílias encontram-se representados por apenas uma espécie cada na arborização urbana de Três Rios (Tabelas 1,2).

Tabela 2. Lista das famílias presentes na arborização urbana de Três Rios, Rio de Janeiro, Brasil e seus parâmetros fitossociológicos.

\begin{tabular}{|c|c|c|c|c|c|c|c|c|c|c|c|c|}
\hline Famílias & NInd & Nam & DR & FA & FR & DoR & Alt. (m) & $\begin{array}{l}\text { Méd. Alt. } \\
(\mathrm{m})\end{array}$ & Diâm. (cm) & $\begin{array}{l}\text { Méd. Diâm. } \\
(\mathrm{cm})\end{array}$ & NSpp & $\%$ Spp \\
\hline Fabaceae & 125 & 5 & 27,6 & 100 & 8,5 & 26,4 & $2,1-14,0$ & $8,1 \pm 3,0$ & $8,0-121,3$ & $32.6 \pm 17,6$ & 10 & 22,2 \\
\hline Combretaceae & 50 & 5 & 11,1 & 100 & 8,5 & 17,3 & $3,0-18,0$ & $9,7 \pm 3,3$ & $19,7 \cdot 76,7$ & $32,8 \pm 17,6$ & 1 & 2,2 \\
\hline Malvaceae & 44 & 4 & 9,7 & 80 & 6,8 & 10 & $2,5-14,5$ & $6,8 \pm 2,7$ & $5,7-80,5$ & $35,9 \pm 14,4$ & 6 & 13,3 \\
\hline Chrysobalanaceae & 39 & 5 & 8,6 & 100 & 8,5 & 17,9 & $1,7-11,0$ & $6,4 \pm 2,1$ & $7,6 \cdot 238,7$ & $41,1 \pm 36,7$ & 1 & 2,2 \\
\hline Rutaceae & 36 & 2 & 8,0 & 40 & 3,4 & 9,2 & $1,5-8,0$ & $4,0 \pm 1,2$ & $6,4-80,5$ & $37,0 \pm 17,5$ & 1 & 2,2 \\
\hline Arecaceae & 33 & 3 & 7,3 & 60 & 5,1 & 3,9 & $3,5-13,0$ & $8,3 \pm 2,7$ & $6,4 \cdot 43,9$ & $25,5 \pm 11,6$ & 3 & 6,7 \\
\hline Bignoniaceae & 31 & 5 & 6,9 & 100 & 8,5 & 2 & $2,5-13,0$ & $6,8 \pm 2,6$ & $4,8-54,1$ & $17,1 \pm 12,0$ & 4 & 8,9 \\
\hline Moraceae & 24 & 5 & 5.3 & 100 & 8.5 & 4.1 & $2,3-10.5$ & $5.3 \pm 2.5$ & $10,5-66,9$ & $30.0 \pm 14,9$ & 2 & 4,4 \\
\hline Myrtaceae & 20 & 4 & 4,4 & 80 & 6,8 & 2,3 & $3,0-10,0$ & $6,2 \pm 1,8$ & $10,2-72,3$ & $24,0 \pm 13,6$ & 4 & 8,9 \\
\hline Anacardiaceae & 16 & 4 & 3.5 & 80 & 6,8 & 2.4 & $3,0-10,0$ & $6,0 \pm 2,1$ & $16,2-51,3$ & $29,4 \pm 10,3$ & 2 & 4,4 \\
\hline Lythraceae & 9 & 4 & 2 & 80 & 6,8 & 0,7 & $3,0 \cdot 6,0$ & $4,5 \pm 1,0$ & $8,3 \cdot 44,3$ & $20,1 \pm 12,2$ & 2 & 4,4 \\
\hline Oleaceae & 7 & 2 & 1,5 & 40 & 3,4 & 0,2 & $3,0-5,0$ & $3,7 \pm 0,8$ & $5,7-20,1$ & $12,5 \pm 6,0$ & 1 & 2,2 \\
\hline Apocynaceae & 6 & 2 & 1,3 & 40 & 3,4 & 2,9 & $4,5 \cdot 8,0$ & $5,4 \pm 1,4$ & $10,5-89,1$ & $50,9 \pm 27,3$ & 2 & 4,4 \\
\hline Malpighiaceae & 3 & 3 & 0,7 & 60 & 5,1 & 0.5 & $3,5-4,5$ & $4,2 \pm 0,6$ & $17,5-39,8$ & $30,8 \pm 11,7$ & 1 & 2,2 \\
\hline Asparagaceae & 3 & 2 & 0,7 & 40 & 3,4 & 0,04 & $4,0 \cdot 4,5$ & $4,2 \pm 0,3$ & $4,8 \cdot 11,5$ & $8,8 \pm 3,6$ & 1 & 2,2 \\
\hline Poligygonaceae & 3 & 1 & 0,7 & 20 & 1,7 & 0,03 & $4,0 \cdot 4,5$ & $4,2 \pm 0,3$ & $4,8 \cdot 12,4$ & $7,4 \pm 4,3$ & 1 & 2,2 \\
\hline Araucariaceae & 1 & 1 & 0,2 & 20 & 1,7 & 0,1 & 7,0 & - & 23,2 & - & 1 & 22 \\
\hline Caricaceae & 1 & 1 & 0,2 & 20 & 1,7 & 0,02 & 5,0 & $\cdot$ & 12,7 & - & 1 & 2,2 \\
\hline Magnoliaceae & 1 & 1 & 0,2 & 20 & 1,7 & 0,02 & 7,0 & - & 10,5 & - & 1 & 2,2 \\
\hline
\end{tabular}

Legenda: NInd - número de indivíduos; Nam - número de amostras; DR - densidade relativa; FA - frequência absoluta; FR - frequência relativa; DoR - dominância relativa; Alt. (m) - altura em metros; Méd. Alt. (m) - média da altura \pm desvio padrão; Diâm. (cm) - diâmetro à altura do peito em centímetros; Méd. Diâm. (cm) - média do diâmetro à altura do peito \pm desvio padrão; NSpp - número de espécies; \%Spp - porcentagem de espécies.

As famílias com maior número de indivíduos foram Fabaceae (125), Combretaceae (50), Malvaceae (44), Chrysobalanaceae (39), Rutaceae (36), Arecaceae (33), Bignoniaceae (31), Moraceae (24), Myrtaceae (20) e Anacardiaceae (16), correspondendo à cerca de 92,6\% dos indivíduos amostrados (Tabela 2). Fabaceae apresentou a maior densidade relativa, devido ao seu grande número de indivíduos, e por estar representada por 10 espécies (Tabelas 1,2). As Famílias Fabaceae, Combretaceae e Chrysobalanaceae apresentam frequência absoluta de 100\%, estando representadas em todos os bairros amostrados (Tabela 2).

Nos dados encontrados na Tabela 3, percebe-se que as espécies Terminalia catappa L. (50 indivíduos) (Combretaceae, amendoeira), Licania tomentosa (Benth.) Fritsch (39) (Chrysobalanaceae, oiti), Pachira aquatica Aubl. (37) (Malvaceae, munguba) e Murraya paniculata (L.) Jack (36) (Rutaceae, murta), apresentaram os maiores números de indivíduos, assim como, valores de densidade relativa e dominância relativa. Terminalia catapa e Licania tomentosa foram encontradas em todos os bairros amostrados. Licania tomentosa é encontrada em praticamente todas as ruas, com exceção da Praça Zaquieu Esmeralda, no bairro Triângulo.

Terminalia catappa conhecida popularmente como amendoeira é largamente utilizada na arborização urbana por apresentar copa larga e cheia, e com isso, oferece um eficiente fornecimento de sombra, além de produzir frutos e sementes nutritivos que atraem a fauna, como por exemplo, pássaros e morcegos (Ivani et al. 2008). Em contrapartida, seu uso em áreas urbanas pode acarretar danos às estruturas públicas, como rachaduras em calçadas consequentes de suas raízes muito desenvolvidas e vigorosas, assim como danos à fiação de rede elétrica devido ao seu grande porte, além da 
alta produção de folhas grandes que podem colaborar para o entupimento de redes de esgoto (Miranda et al. 2015).

Murraya paniculata é frequentemente utilizada na arborização urbana por não ocasionar danos às calçadas e fiação, porém, seu uso na arborização é bastante discutido em virtude de ser potencial hospedeiro de Diaphorina citri (Kuwayama, 1908) (Hemiptera: Leviidae), um transmissor de bactérias associadas à praga "huanglongbing" (HLB), que constitui uma das principais ameaças à produção de cítricos no Brasil (Pogetto, 2018). 0 que leva a proibição de sua produção e plantio em São Paulo e Paraná, através de mecanismos legais, como a Instrução Normativa SDA no 10/2005 (Brasil, 2005) e a Lei no 15.953/2008 (Paraná, 2008).

Milano e Dalcin (2000) recomendam que as espécies não ultrapassem o total de 10 a 15\% de indivíduos na arborização de forma a se garantir a diversidade de espécies, porém, esses índices não são seguidos na arborização da cidade de Três Rios, uma vez que, dentre as 45 espécies encontradas, apenas Terminalia catappa apresenta o percentual de $(11,1 \%)$, seguida de Licania tomentosa $(8,6 \%)$ e Pachira aquatica $(8,2 \%)$, enquanto que outras 20 espécies apresentaram um percentual abaixo de $1 \%$, variando entre $0,2 \%$ e 0,8\% (Tabela 3). Conforme Silva (2000), apesar da indicação de manter a biodiversidade mesmo em ambientes urbanos, geralmente é visto na arborização urbana do Brasil, que poucas espécies representam a grande parte da arborização, como é evidenciado também em Três Rios.

Tabela 3. Lista das espécies presentes na arborização urbana de Três Rios, Rio de Janeiro, Brasil e seus parâmetros fitossociológicos.

\begin{tabular}{|c|c|c|c|c|c|c|c|c|c|c|}
\hline Espécies & NInd & DR & NAm & FA & FR & DoR & $\begin{array}{l}\text { Alt. } \\
(\mathrm{m})\end{array}$ & $\begin{array}{l}\text { Méd. } \\
\text { Alt. (m) }\end{array}$ & $\begin{array}{l}\text { Diâm. } \\
\text { (cm) }\end{array}$ & $\begin{array}{l}\text { Méd. } \\
\text { Diâm. } \\
\text { (cm) }\end{array}$ \\
\hline Terminalia catappa L. & 50 & 11,1 & 5 & 100 & 5 & 17,4 & $\begin{array}{l}3,0- \\
18,0\end{array}$ & $9,7 \pm 3,3$ & $\begin{array}{l}19,7- \\
76,7 \\
\end{array}$ & $\begin{array}{c}45,8 \pm \\
13,2 \\
\end{array}$ \\
\hline $\begin{array}{l}\text { Licania tomentosa } \\
\text { (Benth.) Fritsch }\end{array}$ & 39 & 8,6 & 5 & 100 & 5 & 17,9 & $\begin{array}{l}1,7- \\
11,0\end{array}$ & $6,4 \pm 2,1$ & $\begin{array}{c}7,6- \\
238,7 \\
\end{array}$ & $\begin{array}{l}41,1 \pm \\
36,7 \\
\end{array}$ \\
\hline Pachira aquatica Aubl. & 37 & 8,2 & 3 & 60 & 3 & 8,2 & $\begin{array}{l}2,5- \\
14,5\end{array}$ & $6,9 \pm 2,8$ & $\begin{array}{l}5,7- \\
62,4\end{array}$ & $\begin{array}{c}35,9 \pm \\
13,1\end{array}$ \\
\hline $\begin{array}{l}\text { Murraya paniculata (L.) } \\
\text { Jack }\end{array}$ & 36 & 8 & 2 & 40 & 2 & 9,2 & $1,5-8,0$ & $4,0 \pm 1,2$ & $\begin{array}{l}6,4- \\
80,5\end{array}$ & $\begin{array}{c}37,0 \pm \\
17,5 \\
\end{array}$ \\
\hline $\begin{array}{l}\text { Leucaena leucocephala } \\
\text { (Lam) De Wit. }\end{array}$ & 27 & 6 & 1 & 20 & 1 & 5 & $\begin{array}{l}2,1- \\
14,0\end{array}$ & $\begin{array}{c}11,5 \pm \\
2,6 \\
\end{array}$ & $\begin{array}{l}18,5- \\
81,8\end{array}$ & $\begin{array}{c}32,2 \pm \\
13,1 \\
\end{array}$ \\
\hline $\begin{array}{l}\text { Syagrus romanzoffiana } \\
\text { (Cham.) Glassman }\end{array}$ & 26 & 5,8 & 3 & 60 & 3 & 3,6 & $\begin{array}{l}3,5- \\
13,0\end{array}$ & $9,0 \pm 2,5$ & $\begin{array}{l}12,4- \\
43,9 \\
\end{array}$ & $\begin{array}{c}27,9 \pm \\
11,1 \\
\end{array}$ \\
\hline $\begin{array}{l}\text { Handroanthus } \\
\text { impetiginosus (Mart. ex } \\
\text { DC.) Mattos }\end{array}$ & 24 & 5,3 & 5 & 100 & 5 & 1,9 & $\begin{array}{l}3,0- \\
13,0\end{array}$ & $7,3 \pm 2,5$ & $\begin{array}{l}4,8- \\
54,1\end{array}$ & $\begin{array}{c}18,7 \pm \\
13,0\end{array}$ \\
\hline Bauhinia variegata $\mathrm{L}$. & 23 & 5,1 & 4 & 80 & 4 & 3,9 & $2,5-9,0$ & $5,3 \pm 1,8$ & $\begin{array}{l}10,2- \\
79,3 \\
\end{array}$ & $\begin{array}{c}28,7 \pm \\
17,0 \\
\end{array}$ \\
\hline Senna siamea Lam. & 22 & 4,9 & 4 & 80 & 4 & 5 & $\begin{array}{l}6,0- \\
12,8 \\
\end{array}$ & $9,2 \pm 2,0$ & $\begin{array}{c}25,8- \\
58,9 \\
\end{array}$ & $37,6 \pm 9,2$ \\
\hline Ficus benjamina $\mathrm{L}$. & 21 & 4,7 & 5 & 100 & 5 & 3,5 & $\begin{array}{l}2,3- \\
10,5\end{array}$ & $5,2 \pm 2,5$ & $\begin{array}{l}10,5- \\
66,9 \\
\end{array}$ & $\begin{array}{c}29,2 \pm \\
15,8 \\
\end{array}$ \\
\hline Cassia fistula L. & 15 & 3,3 & 3 & 60 & 3 & 0,9 & $\begin{array}{l}3,0- \\
12,0\end{array}$ & $6,7 \pm 2,0$ & $\begin{array}{l}8,0- \\
33,1\end{array}$ & $18,5 \pm 6,5$ \\
\hline Mangifera indica L. & 14 & 3,1 & 3 & 60 & 3 & 2 & $\begin{array}{l}4,0- \\
10,0\end{array}$ & $6,3 \pm 2,0$ & $\begin{array}{l}16,2- \\
51,3 \\
\end{array}$ & $\begin{array}{c}28,7 \pm \\
10,9 \\
\end{array}$ \\
\hline
\end{tabular}


Tabela 3. Continuação.

\begin{tabular}{|c|c|c|c|c|c|c|c|c|c|c|}
\hline Espécies & NInd & DR & NAm & FA & FR & DoR & $\begin{array}{l}\text { Alt. } \\
\text { (m) }\end{array}$ & $\begin{array}{c}\text { Méd. } \\
\text { Alt. (m) }\end{array}$ & $\begin{array}{l}\text { Diâm. } \\
\text { (cm) }\end{array}$ & $\begin{array}{l}\text { Méd. } \\
\text { Diâm. } \\
\text { (cm) }\end{array}$ \\
\hline $\begin{array}{l}\text { Bauhinia variegata var. } \\
\text { candida Voigt }\end{array}$ & 11 & 2,4 & 4 & 80 & 4 & 1,1 & $4,0-8,0$ & $6,0 \pm 1,2$ & $\begin{array}{l}11,8- \\
38,2\end{array}$ & $23,8 \pm 9,1$ \\
\hline $\begin{array}{l}\text { Delonix regia (Bojer ex } \\
\text { Hook.) Raf. (Bojer ex } \\
\text { Hook.) Raf. }\end{array}$ & 9 & 2 & 3 & 60 & 3 & 3,1 & $4,0-9,0$ & $6,7 \pm 1,6$ & $\begin{array}{l}12,1- \\
77,0\end{array}$ & $\begin{array}{c}43,0 \pm \\
22,0\end{array}$ \\
\hline $\begin{array}{ll}\text { Peltophorum } & \text { dubium } \\
\text { (Spreng.) Taub. } & \\
\end{array}$ & 8 & 1,8 & 2 & 40 & 2 & 3,2 & $\begin{array}{l}4,0- \\
12,0 \\
\end{array}$ & $8,6 \pm 3,0$ & $\begin{array}{c}20,4- \\
88,2 \\
\end{array}$ & $\begin{array}{c}45,0 \pm \\
25,7 \\
\end{array}$ \\
\hline Lagerstroemia indica L. & 8 & 1,8 & 4 & 80 & 4 & 0,7 & $3,0-6,0$ & $4,4 \pm 1,1$ & $\begin{array}{l}8,3- \\
44,3\end{array}$ & $\begin{array}{c}20,5 \pm \\
13,0\end{array}$ \\
\hline Albizia lebbeck (L.) Benth & 7 & 1,6 & 3 & 60 & 3 & 1,4 & $\begin{array}{l}6,0- \\
12,0 \\
\end{array}$ & $7,8 \pm 2,0$ & $\begin{array}{c}18,5- \\
51,9 \\
\end{array}$ & $\begin{array}{c}34,3 \pm \\
12,5 \\
\end{array}$ \\
\hline Psidium guajava L. & 7 & 1,6 & 3 & 60 & 3 & 0,3 & $4,5-7,5$ & $5,3 \pm 0,9$ & $\begin{array}{l}10,2- \\
24,2\end{array}$ & $16,1 \pm 4,4$ \\
\hline $\begin{array}{l}\text { Ligustrum lucidum W. T. } \\
\text { Aiton }\end{array}$ & 7 & 1,6 & 2 & 40 & 2 & 0,2 & $3,0-5,0$ & $3,7 \pm 0,8$ & $\begin{array}{l}5,7- \\
20,1 \\
\end{array}$ & $12,5 \pm 6,0$ \\
\hline $\begin{array}{lll}\text { Syzygium } & \text { cumini } & \text { (L.) } \\
\text { Skeels } & & \\
\end{array}$ & 7 & 1,6 & 1 & 20 & 1 & 0,7 & $5,5-9,0$ & $6,5 \pm 1,3$ & $\begin{array}{l}13,4- \\
37,9\end{array}$ & $24,1 \pm 8,5$ \\
\hline Nerium oleander L. & 5 & 1,1 & 2 & 40 & 2 & 1,7 & $4,5-8,0$ & $5,6 \pm 1,5$ & $\begin{array}{l}10,5- \\
71,0\end{array}$ & $\begin{array}{c}43,2 \pm \\
22,2\end{array}$ \\
\hline $\begin{array}{lll}\text { Syzygium jambos } & \text { (L.) } \\
\text { Alston } & & \\
\end{array}$ & 5 & 1,1 & 3 & 60 & 3 & 0,5 & $\begin{array}{l}3,0- \\
10,0 \\
\end{array}$ & $7,2 \pm 3,0$ & $\begin{array}{l}16,6- \\
32,8\end{array}$ & $25,5 \pm 7,1$ \\
\hline $\begin{array}{l}\text { Socratea exorrhiza } \\
\text { (Mart.) H. Wendl. }\end{array}$ & 4 & 0,9 & 1 & 20 & 1 & 0,1 & $4,0-5,0$ & $4,5 \pm 0,6$ & $\begin{array}{l}6,4- \\
12,4 \\
\end{array}$ & $9,3 \pm 2,5$ \\
\hline $\begin{array}{l}\text { Malpighia emarginata } \\
\text { DC. }\end{array}$ & 3 & 0,7 & 3 & 60 & 3 & 0,5 & $3,5-4,5$ & $4,2 \pm 0,6$ & $\begin{array}{l}17,5- \\
39,8 \\
\end{array}$ & $\begin{array}{c}30,8 \pm \\
11,7 \\
\end{array}$ \\
\hline Cocos nucifera L. & 3 & 0,7 & 2 & 40 & 2 & 0,3 & $6,0-9,0$ & $7,3 \pm 1,5$ & $\begin{array}{c}24,2- \\
27,1\end{array}$ & $25,5 \pm 1,5$ \\
\hline $\begin{array}{l}\text { Tecoma stans (L.) Juss. ex } \\
\text { Kunth }\end{array}$ & 3 & 0,7 & 2 & 40 & 2 & 0,1 & $2,5-7,0$ & $4,8 \pm 2,3$ & $\begin{array}{l}5,7- \\
22,0\end{array}$ & $12,8 \pm 8,3$ \\
\hline $\begin{array}{l}\text { Handroanthus } \\
\text { chrysotrichus (Mart. ex } \\
\text { DC.) Mattos }\end{array}$ & 3 & 0,7 & 2 & 40 & 2 & 0,05 & $3,5-5,0$ & $4,2 \pm 0,8$ & $\begin{array}{l}9,2- \\
11,1\end{array}$ & $10,3 \pm 1,0$ \\
\hline $\begin{array}{l}\text { Dracaena fragrans (L.) } \\
\text { Ker Gawl. }\end{array}$ & 3 & 0,7 & 2 & 40 & 2 & 0,04 & $4,0-4,5$ & $4,2 \pm 0,3$ & $\begin{array}{l}4,8- \\
11,5 \\
\end{array}$ & $8,8 \pm 3,6$ \\
\hline Morus nigra L. & 3 & 0,7 & 1 & 20 & 1 & 0,6 & $3,0-9,0$ & $6,3 \pm 3,1$ & $\begin{array}{c}29,9- \\
40,7\end{array}$ & $35,2 \pm 5,4$ \\
\hline $\begin{array}{l}\text { Ceiba speciosa (A. St.-Hil.) } \\
\text { Ravenna }\end{array}$ & 3 & 0,7 & 1 & 20 & 1 & 0,5 & $8,0-9,0$ & $8,3 \pm 0,6$ & $\begin{array}{c}30,9- \\
36,0\end{array}$ & $34,1 \pm 2,8$ \\
\hline Triplaris americana $\mathrm{L}$. & 3 & 0,7 & 1 & 20 & 1 & 0,03 & $4,0-4,5$ & $4,2 \pm 0,3$ & $\begin{array}{l}4,8- \\
12,4\end{array}$ & $7,4 \pm 4,3$ \\
\hline $\begin{array}{l}\text { Clitoria fairchildiana R. A. } \\
\text { Howard }\end{array}$ & 2 & 0,4 & 1 & 20 & 1 & 2,5 & $\begin{array}{l}6,5- \\
10,5 \\
\end{array}$ & 8,5 & $\begin{array}{l}36,9- \\
121,3 \\
\end{array}$ & 79,1 \\
\hline $\begin{array}{l}\text { Schinus } \\
\text { Raddi }\end{array}$ & 2 & 0,4 & 2 & 40 & 2 & 0,4 & $3,0-4,0$ & 3,5 & $\begin{array}{c}33,7- \\
34,4\end{array}$ & 34,1 \\
\hline Plumeria rubra L. & 1 & 0,2 & 1 & 20 & 1 & 1,2 & 4,5 & & 89,1 & \\
\hline $\begin{array}{ll}\text { Ochroma pyramidale } \\
\text { (Cav. ex Lam.) Urb. }\end{array}$ & 1 & 0,2 & 1 & 20 & 1 & 1 & 4,0 & & 80,5 & \\
\hline $\begin{array}{l}\text { Plinia cauliflora (Mart.) } \\
\text { Kausel }\end{array}$ & 1 & 0,2 & 1 & 20 & 1 & 0,8 & 6,0 & & 72,3 & \\
\hline Bauhinia blakeana Dunn & 1 & 0,2 & 1 & 20 & 1 & 0,4 & 6,0 & & 49,0 & \\
\hline
\end{tabular}


Tabela 3. Continução.

\begin{tabular}{|c|c|c|c|c|c|c|c|c|c|c|}
\hline Espécies & NInd & DR & NAm & FA & FR & DoR & $\begin{array}{l}\text { Alt. } \\
\text { (m) }\end{array}$ & $\begin{array}{c}\text { Méd. } \\
\text { Alt. (m) }\end{array}$ & $\begin{array}{l}\text { Diâm. } \\
\text { (cm) }\end{array}$ & $\begin{array}{l}\text { Méd. } \\
\text { Diâm. } \\
\text { (cm) }\end{array}$ \\
\hline Guazuma ulmifolia Lam. & 1 & 0,2 & 1 & 20 & 1 & 0,1 & 5,5 & & 29,0 & \\
\hline Dombeya acutangula Cav. & 1 & 0,2 & 1 & 20 & 1 & 0,1 & 7,0 & & 24,5 & \\
\hline $\begin{array}{l}\text { Araucaria columnaris (G. } \\
\text { Forst.) Hook }\end{array}$ & 1 & 0,2 & 1 & 20 & 1 & 0,1 & 7,0 & & 23,2 & \\
\hline $\begin{array}{l}\text { Physocalymma } \\
\text { scaberrimum Pohl. }\end{array}$ & 1 & 0,2 & 1 & 20 & 1 & 0,05 & 5,0 & & 17,2 & \\
\hline Hibiscus rosa-sinensis L. & 1 & 0,2 & 1 & 20 & 1 & 0,03 & 4,0 & & 14,0 & \\
\hline Carica papaya L. & 1 & 0,2 & 1 & 20 & 1 & 0,02 & 5,5 & & 12,7 & \\
\hline $\begin{array}{l}\text { Magnolia champaca (L.) } \\
\text { Baill. ex Pierre }\end{array}$ & 1 & 0,2 & 1 & 20 & 1 & 0,02 & 7,0 & & 10,1 & \\
\hline $\begin{array}{l}\text { Handroanthus } \\
\text { serratifolius (Vahl) S. } \\
\text { Grose }\end{array}$ & 1 & 0,2 & 1 & 20 & 1 & 0,01 & 8,0 & & 9,9 & \\
\hline
\end{tabular}

Legenda: NInd - número de indivíduos; Nam - número de amostras; DR - densidade relativa; FA frequência absoluta; FR - frequência relativa; DoR - dominância relativa; Alt. (m) - altura em metros; Méd. Alt. (m) - média da altura \pm desvio padrão; Diâm. $(\mathrm{cm})$ - diâmetro à altura do peito em centímetros; Méd. Diâm. (cm) - média do diâmetro à altura do peito \pm desvio padrão.

De acordo com a relação total do número de espécies e indivíduos amostrados, o Coeficiente de Mistura de Jentsch (QM) encontrado foi de 0,1 ou 1:10, o que indica uma média de dez indivíduos para cada espécie, porém a maioria dos indivíduos estão concentrados em apenas 13 espécies, e dez são representadas por apenas uma espécie (Tabela 3).

Faria et al. (2013) e Bastos et al. (2016), nos quais analisaram ruas dos bairros Centro e Vila Isabel, somaram a este trabalho mais 14 espécies (Tabela 4). Destas, Cenostigma pluviosum var. peltophoroides (Benth.) E. Gagnon \& G.P. Lewis (Fabaceae sibipiruna) com 16 indivíduos, Pleroma granulosum (Desr.) D. Don (Melastomataceae quaresminha) com um indivíduo e Sapindus saponaria L. (Sapindaceae - sabão de soldado) com um indivíduo no bairro Centro, são representantes de espécies nativas da Mata Atlântica e ocorrem no estado do Rio de Janeiro.

Cenostigma pluviosum var. peltophoroides e Pleroma granulosum são endêmicas do estado do Rio de Janeiro, configurando espécies importantes para estar entre os representantes da arborização urbana de Três Rios. As demais espécies são exóticas do Brasil. Com este acréscimo, a arborização urbana de Três Rios conta com 59 espécies.

A partir do cálculo de indivíduos por extensão de calçadas em metros, infere-se que todos os bairros analisados apresentaram número de indivíduos a cada $10 \mathrm{~m}$ abaixo de um, o que possibilita caracterizar a arborização das vias como descontínua e desordenada, visto que na maioria dos casos havia áreas onde os indivíduos arbóreos estavam muito próximos e em outras áreas decorriam-se grandes distâncias sem arborização (Tabela 5). 
Tabela 4. Lista das espécies presentes na arborização urbana de Três Rios, Rio de Janeiro, Brasil, retirados de Faria et al. (2013) e Bastos et al. (2016), para os bairros Centro e Vila Isabel.

\begin{tabular}{|c|c|c|c|}
\hline Família & Espécie & Nome popular & Origem \\
\hline \multirow[t]{2}{*}{ Anacardiaceae } & Spondias purpurea L. & Siriguela & EX-BR \\
\hline & Spondias dulcis Parkinson* & Cajá-manga & EX-BR \\
\hline Annonaceae & Cananga odorata Lam. & $\begin{array}{l}\text { Cananga, } \\
\text { Ilangue-ilangue }\end{array}$ & EX-BR \\
\hline Arecaceae & Roystonea oleraceae (Jacq.) O. F. Cook & Palmeira & EX-BR \\
\hline \multirow[t]{2}{*}{ Bignoniaceae } & Tabebuia pentaphylla (L.) Hemsl. & Ipê-rosa & EX-BR \\
\hline & Spathodea campanulata P. Beauv. & Tulipeira & EX-BR \\
\hline Fabaceae & $\begin{array}{l}\text { Cenostigma pluviosum var. } \\
\text { peltophoroides (Benth.) E. Gagnon \& G. } \\
\text { P. Lewis* }\end{array}$ & Sibipiruna & NMA \\
\hline Lythraceae & Punica granatum L. & Romã & EX-BR \\
\hline Malpighiaceae & Malpighia glabra L.* & Acerola & EX-BR \\
\hline \multirow[t]{2}{*}{ Melastomataceae } & Tibouchina adenophora Cogn. & Quaresminha & EX-BR \\
\hline & Pleroma granulosum (Desr.) D. Don & Quaresminha & NMA \\
\hline Oxalidaceae & Averrhoa carambola L. & Carambola & EX-BR \\
\hline Rosaceae & Prunus sp. & Ameixeira & EX-BR \\
\hline Sapindaceae & Sapindus saponaria L.* & Sabão-de-soldado & NBR/NMA \\
\hline
\end{tabular}

Legenda: Origem - EX-BR: exótica do Brasil, EX-MA: exótica da Mata Atlântica, NBR: nativa do Brasil, NMA: nativa da Mata Atlântica; *espécies encontradas no levantamento florístico da mata ciliar urbana do Rio Paraíba do Sul no Município de Três Rios (Candido, 2015; Candido et al., 2018).

Tabela 5. Análise de densidade de indivíduos arbóreos por metro de calçada na arborização urbana do Município de Três Rios, Rio de Janeiro, Brasil.

\begin{tabular}{|l|c|c|c|}
\hline Bairro & $\begin{array}{c}\text { Extensão total de } \\
\text { calçadas (m) }\end{array}$ & $\begin{array}{c}\text { No de } \\
\text { indivíduos }\end{array}$ & モ Árvore $/ \mathbf{1 0 m}$ \\
\hline Cantagalo & 2.500 & 120 & $0,5=1$ árvore $/ 21 \mathrm{~m}$ \\
\hline Centro & 3.060 & 123 & $0,4=1$ árvore $/ 25 \mathrm{~m}$ \\
\hline Nova Niterói & 3.685 & 137 & $0,4=1$ árvore $/ 27 \mathrm{~m}$ \\
\hline Palmital & 1.440 & 37 & $0,3=1$ árvore $/ 39 \mathrm{~m}$ \\
\hline Triângulo & 2.574 & 35 & $0,1=1$ árvore $/ 74 \mathrm{~m}$ \\
\hline Centro (Bastos et al. 2016) & 6.000 & 310 & $0,5=1$ árvore $/ 19 \mathrm{~m}$ \\
\hline Centro (Farias et al. 2013) & 2.272 & 139 & $0,6=1$ árvore $/ 16 \mathrm{~m}$ \\
\hline Vila Isabel (Farias et al. 2013) & 1.975 & 9 & $0,05=1$ árvore $/ 219 \mathrm{~m}$ \\
\hline
\end{tabular}

É perceptível a diferença na composição da arborização a partir da identidade dos bairros, pois bairros mais valorizados, como Centro, Nova Niterói e Cantagalo apresentaram ruas mais arborizadas com 123, 137 e 120 indivíduos, respectivamente, apresentando uma árvore a cada 16 a $27 \mathrm{~m}$ de distância (Tabela 5), enquanto que Palmital, Triângulo e Vila Isabel que são bairros tidos como mais periféricos apresentaram uma quantidade reduzida de indivíduos na arborização com 37, 35 e nove, respectivamente. 0 bairro Vila Isabel demonstrou a menor arborização urbana, segundo Faria et al. (2013), exibindo uma árvore a cada $219 \mathrm{~m}$ de calçada.

Em relação à estrutura vertical da arborização urbana de Três Rios, os indivíduos amostrados apresentam alturas entre 1,5 e 18,0 $\mathrm{m}$, com média de 7,0 $\mathrm{m} \pm 3,0 \mathrm{~m}$ de desvio padrão (Tabelas 2 e 3). Existem alguns indivíduos acima da altura média, como por 
exemplo, um indivíduo de Terminalia catappa de altura estimada de $18 \mathrm{~m}$, presente no Bairro Centro, Rua Barão de Entre Rios.

Já o diâmetro na altura do peito (DAP) variou de 4,8 a 238,7 cm, com diâmetro médio de $32,5 \mathrm{~cm} \pm 19,8 \mathrm{~cm}$ de desvio padrão, o DAP máximo foi observado para um indivíduo de Licania tomentosa, presente no Bairro Cantagalo, Rua Benjamin Constant (Tabelas 2 e 3).

Foi constatado que 38\% dos indivíduos amostrados apresentam interferência na rede elétrica, visto que, grande parte das espécies utilizadas é de grande porte, atingindo assim, a fiação. Dentre os problemas ocasionados pelo conflito entre árvore e rede elétrica, podem ser observados, os rompimentos de cabos, curtos-circuitos, interrupções no fornecimento de energia e queima de eletrodomésticos, como notado por Carneiro e Carvalho (2013).

Em contrapartida, 41\% dos indivíduos estavam em locais onde não havia presença de rede elétrica, apresentando melhor adequação ao ambiente urbano e oferecendo menos riscos. E os $21 \%$ restantes foram espécies que estavam localizadas onde havia a presença da rede elétrica, porém não apresentavam interferência na rede, pois não alcançavam a altura da fiação.

Observou-se também que $29,7 \%$ das árvores levantadas apresentaram interferência na calçada, apresentando raízes expostas que causam rachaduras nos passeios, e oferecem riscos aos transeuntes, além de impedir a passagem de pessoas com mobilidade reduzida. Este fato é devido à falta de adequação no plantio das árvores, visto que, na maioria dos casos, o sistema radicular dos indivíduos encontrava-se totalmente cimentado, não oferecendo uma área livre permeável para o desenvolvimento das raízes.

A grande ocorrência de conflitos entre indivíduos arbóreos e estruturas urbanas indica a falta de planejamento da arborização. 0 não planejamento da arborização acarreta em problemas e prejuízos no pavimento das calçadas e muros, dificultando a mobilidade de portadores de deficiência, rompimento da rede elétrica, entre outros. 0 que foi evidenciado em todos os bairros analisados.

Além disso, a arborização mal planejada dificulta a circulação de pedestres e/ou veículos quando são utilizadas espécies com galhos laterais muito baixos, e também, podem causar o entupimento de encanamentos pluviais, no uso de espécies com folhas decíduas ou quando a biomassa vegetal não é recolhida eficientemente pelo serviço de limpeza pública, o que pode aumentar o risco de enchentes (Christo e Dias, 2007).

Por estes motivos, a recomendação é que se utilizem espécies de porte adequado e espécies nativas, pois as espécies nativas promovem a atração da avifauna local, e a introdução de espécies exóticas invasoras pode resultar na invasão biológica ou contaminação biológica de remanescentes florestais naturais adjacentes as cidades (Sampaio et al., 2011; Santos et al., 2013).

Das espécies inventariadas na arborização urbana de Três Rios, 19 (42\%) foram encontradas no levantamento florístico da mata ciliar urbana do rio Paraíba do Sul no município de Três Rios, sendo 12 (27\%) exóticas e sete nativas brasileiras (Candido, 2015; Candido et al., 2018), este resultado demonstra que o uso indevido de plantas exóticas na arborização urbana, pode influenciar no acréscimo dessas plantas em áreas de resquícios de fragmentos de vegetação secundária.

Assim como no presente trabalho, Faria et al. (2013) e Bastos et al. (2016), também constataram que a maioria das espécies utilizadas na arborização urbana de Três Rios são exóticas aos ecossistemas brasileiros, demonstrando que a arborização das vias públicas na cidade não foi bem planejada, já que essas espécies se proliferam sem controle e representam uma ameaça para espécies nativas, gerando desequilíbrio no ecossistema e reduzindo a diversidade da região.

As espécies Dracaena fragrans (L.) Ker Gawl., Leucaena leucocephala (Lam.) De Wit e Terminalia catappa aparecem na lista de espécies invasoras no município do Rio de 
Janeiro, de acordo com a Resolução SMAC no 492/2011 (Rio de Janeiro, 2011), que regulamenta o Programa Municipal de Controle de Espécies Invasoras Vegetais. Recentemente, o CNCFlora (2018) publicou uma listagem de plantas alternativas às plantas exóticas invasoras listadas para o Estado do Rio de Janeiro, informando plantas nativas com as mesmas características morfológicas, ou características similares das exóticas, demonstrando a importância de se realizar um manejo adequado para que não haja perda da biodiversidade.

O devido planejamento concordando características das espécies arbóreas e equipamentos públicos assegura um adequado desenvolvimento das árvores, previne acidentes e facilita o manejo da arborização (Prefeitura de São Paulo, 2015), assim como, é capaz de oferecer uma ampliação na qualidade do meio e redução dos impactos decorrentes do processo de urbanização, aumentando as condições estéticas, paisagísticas e de potencial ecológico (Melo e Piacentini, 2011).

0 grande desafio para arborização brasileira é a valorização da biodiversidade local, pois dessa maneira as florestas urbanas podem servir como um instrumento para conservação da diversidade, então propostas de retirada de grande parte das árvores exóticas ou invasoras existentes e o plantio de novas espécies nativas estão sendo almejadas, como uma forma de manutenção da biodiversidade e atração da fauna local em busca de alimento e habitat (Pinheiro et al., 2009).

0 problema é que a escolha das espécies arbóreas usadas na arborização urbana é pouco criteriosa, devido a baixa oferta de mudas em viveiros, a ausência de profissionais com capacitação técnica em prefeituras e os poucos estudos sobre a flora regional (Santos et al., 2013).

\section{Conclusão}

Três Rios não possui um planejamento urbano que aborde a arborização, visto que o inventário demonstrou uma grande proporção de espécies exóticas (62\%) que sobrepõe à proporção de espécies nativas (38\%), além de apresentarem-se de forma descontínua e desordenada nos bairros analisados, visto que na maioria dos casos havia áreas onde os indivíduos arbóreos estavam muito próximos e em outras áreas decorriam-se grandes distâncias sem arborização. Outro fator que também demonstra a falta de planejamento foi à incompatibilidade com as estruturas urbanas, como pavimento e rede elétrica, onde grande parte dos indivíduos arbóreos encontrou-se em conflito, oferecendo riscos à população.

Sendo assim, é necessária uma maior atenção ao aumento da diversidade de espécies na arborização do município, visto que as ruas analisadas apresentaram em sua composição, a predominância de poucas espécies como Terminalia catappa, Licania tomentosa e Pachira aquatica, enquanto que outras espécies apresentaram um número reduzido de indivíduos, como o caso de Triplaris americana L., Schinus terebinthifolia Raddi, Handroanthus chrysotrichus (Mart. ex DC.) Mattos que são espécies nativas e por isso seriam mais adequadas para utilização na arborização urbana.

Sugere-se então, a realização de um planejamento para a arborização do município, incluindo avaliação dos indivíduos arbóreos já existentes, realização de possíveis adequações às estruturas urbanas como podas e abertura de canteiros, de forma que seja respeitada uma área permeável para o sistema radicular das árvores, assim como o desenvolvimento de técnicas de manutenção desses indivíduos e plantio de novas árvores. Além disso, mostra-se relevante também uma reavaliação da arborização em bairros mais periféricos, como Palmital e Triângulo que quando comparados com os bairros mais valorizados, como o Centro, demonstra uma arborização deficiente, com um número de indivíduos reduzido e uma baixa diversidade de espécies. 


\section{Agradecimentos}

Ao PIBIC/CNPq/UFRRJ pela bolsa de Iniciação Científica concedida à primeira autora, à Universidade Federal Rural do Rio de Janeiro pela infraestrutura, à Thayná Ribeiro Bromana pela produção do mapa.

\section{Conflito de interesses}

As autoras declaram não haver conflito de interesses.

\section{Referências}

APG IV - Angiosperm Phylogeny Group. An update of the Angiosperm Phylogeny Group classification for the orders and families of flowering plants: APG IV. Botanical Journal of the Linnean Society, v. 181, n. 1, p. 1-20, 2016. https://doi.org/10.1111/boj.12385

Bastos, A. S. A.; Vieira, B. B.; Amaral, G. S.; Carvalho Júnior, L.; Cruz, V. O. R.; Milward-deAzevedo, M. A. Percepção da arborização urbana na Cidade de Três Rios, RJ. In: Milwardde-Azevedo, M. A.; Cortines, E. (Eds.). Anais do 5으 Simpósio de Gestão Ambiental e Biodiversidade. Três Rios, 2016, p. 246-253. Disponível em: <https://itr.ufrrj.br/ sigabi/wp-content/uploads/5_sigabi/Sumarizado/35.pdf>. Acesso em: 17 jun. 2019.

Blum, C. T.; Borgo, M.; Sampaio, A. C. F. Espécies exóticas invasoras na arborização de vias públicas de Maringá-PR. Revista Brasileira de Arborização Urbana, v. 3, n. 2, p. 78-97, 2008. https://doi.org/10.5380/revsbau.v3i2.66347

Brasil. Instrução Normativa SDA no 10, 18 de março de 2005. Da continuidade aos trabalhos de levantamento da ocorrência do HLB, visando a delimitar a extensão das áreas afetadas e adotar medidas de prevenção e erradicação. Disponível em: <https://www.diariodasleis.com.br/busca/exibelink.php?numlink=1-101-23-2005-0318-10>. Acesso em: 23 jul. 2020.

Candido, H. M. N. Florística e fitossociologia em mata ripária como instrumento de planejamento e conservação da biodiversidade em Três Rios, RJ. Três Rios: Universidade Federal Rural do Rio de Janeiro, 2015. (Trabalho de Conclusão de Curso).

Candido, H. M. N.; Pereira, A. L.; Cortines, E.; Milward-de-Azevedo, M. A. Censo florestal de um trecho de mata ciliar urbana do Rio Paraíba do Sul, Três Rios, RJ. Diversidade e Gestão, v. 2, n. 1, p. 17-25, 2018.

Carneiro, D. C.; Carvalho, S. M. As estruturas de gestão e planejamento da arborização urbana de Ponta Grossa, PR. Periódico Técnico e Científico Cidades Verdes, v. 1, n. 1, p. 94-116, 2013. https://doi.org/10.17271/23178604112013412

Christo, J. A.; Dias, A. N. Inventário florestal da arborização urbana do centro da Cidade de Prudentópolis, PR. Revista Eletrônica Lato Sensu, v. 2, p. 76-93, 2007.

CNCFlora - Centro Nacional de Conservação da Flora. Listagem de plantas alternativas às plantas exóticas invasoras listadas para o Estado do Rio de Janeiro. Rio de Janeiro: Jardim Botânico do Rio de Janeiro, 2018. Disponível em: <https://ckan.jbrj.gov.br/ dataset/edit/plantasalternativas-as-plantas-exoticas-invasoras-rj>. Acesso em: 15 jun. 2019. 
Faria, D. C.; Duarte, J. M. A.; Pinto, D. M.; Almeida, F. S. Arborização urbana no Município de Três Rios-RJ: espécies utilizadas e a percepção de seus benefícios pela população. Revista da Sociedade Brasileira de Arborização Urbana, v. 8, n. 2, p. 58-67, 2013. https://doi.org/10.5380/revsbau.v8i2.66422

Faria, J. L. G.; Monteiro, E. A.; Fisch, S. T. V. Arborização de vias públicas do Município de Jacareí, SP. Revista da Sociedade Brasileira de Arborização Urbana, v. 2, n. 4, p. 20-33, 2007. https://doi.org/10.5380/revsbau.v2i4.66335

Graciano-Silva, T.; Cardoso-Leite, E.; Tonello, K. C. Inventário da arborização urbana no Município de Araçoiaba da Serra, SP. Revista Brasileira da Sociedade de Arborização Urbana, v. 9, n. 4, p. 151-169, 2014. https://doi.org/10.5380/revsbau.v9i4.63322

Hosokawa, R. T. Manejo de florestas tropicais úmidas em regime de rendimento sustentado. Curitiba: CNPq/IBDF/UFPr, 1981.

IBGE - Instituto Brasileiro de Geografia e Estatística. Censo demográfico: Rio de Janeiro. 2014. Disponível em: <ftp://ftp.ibge.gov.br/Estimativas_de_Populacao/Estimativas_2014/ estimativa_dou_2014.pdf >. Acesso em: 18 mar. 2019.

IBGE - Instituto Brasileiro de Geografia e Estatística. Manual técnico da vegetação brasileira. Brasília: IBGE, 2012. Disponível em: <ftp://geoftp.ibge.gov.br/documentos/ recursos_naturais/manuais_tecnicos/manual_tecnico_vegetacao_brasileira.pdf $>$. Acesso em: 18 mar. 2019.

Ivani, S. A.; Silva, B. M. S.; Oliveira, C.; Môro, F. V. Morfologia de frutos, sementes e plântulas de castanheira (Terminalia catappa L. - Combretaceae). Revista Brasileira de

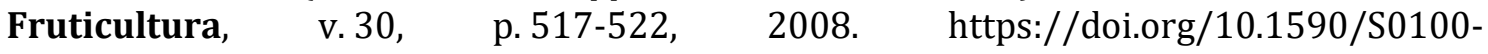
29452008000200043

Iwama, A. Y. Indicador de arborização urbana como apoio ao planejamento de cidades brasileiras. Revista da Sociedade Brasileira de Arborização Urbana, v. 9, n. 3, p. 156-172, 2014. https://doi.org/10.5380/revsbau.v9i3.63121

Melo, E. F. R. Q.; Piacentini, C. A. M. Diversidade da arborização urbana no Município de Colorado (RS). Ambiência, v. 7, n. 2, p. 339-352, 2011.

Milano, M. S.; Dalcin, E. Arborização de vias públicas. Rio de Janeiro: Light, 2000.

Miranda, Y. C.; Machado, M. S.; Silva, L. S.; Estevam, R.; Martins Neto, F. F.; Caxambu, M. G. Análise quali-quantitativa da arborização de ruas do Município de Godoy Moreira, PR. Revista Brasileira da Sociedade de Arborização Urbana, v. 10, n. 1, p. 71-81, 2015. https://doi.org/10.5380/revsbau.v10i1.63210

Mueller-Dombois, D.; Ellenberg, H. Aims and methods of vegetation ecology. New York: John Wiley \& Sons, 1974.

Paraná. Lei no 15.953, 24 de setembro de 2008. Proíbe o plantio, comércio, transporte e produção da planta murta (Murraya paniculata), por ser vegetal hospedeiro da bactéria Candidatus liberibacter ssp., disseminada pelo inseto vetor Diaphorina citri, transmissor da praga denominada huanglongbing (HLB - greening). Disponível em: <http://portal. assembleia.pr.leg.br/index.php/pesquisa-legislativa/legislacao-estadual?idLegislacao= 28448\&tpLei=0\&idProposicao=16583 > . Acesso em: 24 abr. 2020.

Pinheiro, R.; Franchin, E.; Ribeiro, R. S.; Wolff, W.; Silva, A. C.; Higuchi, P. Arborização urbana na Cidade de São José do Cerrito, SC: diagnóstico e proposta para áreas de maior trânsito. Revista da Sociedade Brasileira de Arborização Urbana, v. 4, n. 4, p. 63-78, 2009. https://doi.org/10.5380/revsbau.v4i4.66449 
Pogetto, L. D. Potencial hospedeiro de duas espécies Murraya para Diaphorina citri (Kuwayama, 1908) (Hemiptera: Leviidae) com vistas à produção de Tamarixia radiata Waterston, 1922 (Hymenoptera: Eulophidae). Piracicaba: Universidade de São Paulo, 2018. (Dissertação de mestrado).

Prefeitura de São Paulo. Manual técnico de arborização urbana. São Paulo: Prefeitura de São Paulo, 2015. Disponível em: <https://www.prefeitura.sp.gov.br/cidade/secretarias/ upload/meio_ambiente/MARBOURB.pdf>. Acesso em: 10 fev. 2019.

Rio de Janeiro. Resolução SMAC no 492, de 05 de julho de 2011. Regulamenta o Programa Municipal de Controle de Espécies Exóticas Invasoras Vegetais. Disponível em: <http://www.rio.rj.gov.br/dlstatic/10112/4571912/4115297/RESSMAC4922011invasor as.pdf>. Acesso em: 23 abr. 2020.

Sampaio, A. C. F.; Ecker, A. E. A.; Maragoni, C. J. M.; Fiorese, L. M. R.; Sordi, E. A. Espécies exóticas invasoras de vias públicas de três bairros de Campo Mourão, PR. Campo Digital, v. 6, n. 1, p. 31-43, 2011.

Santos, E. M.; Silveira, B. D. B.; Souza, A. C.; Schmitz, V.; Silva, A. C.; Higuchi, P. Análise quali-quantitativa da arborização urbana em Lages, SC. Revista de Ciências Agroveterinárias, v. 12, n. 1, p. 59-67, 2013.

Shepherd, G. J. Fitopac 2. Campinas: Departamento de Biologia Vegetal, Universidade Estadual de Campinas, 2009.

Silva, A. G. Arborização urbana em cidades de pequeno porte: avaliação quantitativa e qualitativa. Viçosa: Universidade Federal de Viçosa, 2000. (Dissertação de mestrado).

Silva-Filho, E. V.; Gomes, O. V. O.; Marques, E. D.; Souza, M. D. C. Influência antrópica nas águas superficiais da Cidade de Três Rios, RJ. Geochimica Brasiliensis, v. 27, n. 1, p. 77-86, 2013. https://doi.org/10.21715/gb.v27i1.344

Três Rios. Lei no 3.906, de 02 de outubro de 2013. Dispõe sobre a revisão do Plano Diretor do Município de Três Rios, modifica a Lei no 2.962 de 10/10/2006 e dá outras providências. Disponível em: <https://cvtr.rj.gov.br/lei-no-3-906-dispoe-sobre-a-revisaodo-plano-diretor-do-municipio-de-tres-rios-modifica-a-lei-no-2-962-de-10-10-2006-e-daoutras-providencias/>. Acesso em: 23 abr. 2020.

Informação da Licença: Este é um artigo Open Access distribuído sob os termos da Licença Creative Commons Attribution, que permite uso irrestrito, distribuição e reprodução em qualquer meio, desde que a obra original seja devidamente citada. 